\title{
molecules
}

ISSN 1420-3049

www.mdpi.com/journal/molecules

Article

\section{Construction of an Isonucleoside on a 2,6-Dioxobicyclo[3.2.0]-heptane Skeleton}

\author{
Yuichi Yoshimura ${ }^{1, *}$, Satoshi Kobayashi ${ }^{1}$, Hitomi Kaneko ${ }^{1}$, Takeshi Suzuki ${ }^{1}$ and \\ Tomozumi Imamichi ${ }^{2}$
}

1 Faculty of Pharmaceutical Sciences, Tohoku Pharmaceutical University, 4-4-1 Komatsushima, Aoba-ku, Sendai 981-8558, Japan; E-Mails: s-kobayashi@fujichemical.co.jp (S.K.); hitomi.f528@gmail.com (H.K.); suzutake19851109@coral.plala.or.jp (T.S.)

2 Laboratory of Human Retrovirology, Leidos Biochemical Research Inc., Frederick National Laboratory for Cancer Research, Frederick, MD 21702, USA; E-Mail: timamichi@mail.nih.gov

* Author to whom correspondence should be addressed; E-Mail: yoshimura@tohoku-pharm.ac.jp; Tel./Fax: +81-22-727-0144.

Academic Editors: Mahesh K. Lakshman and Fumi Nagatsugi

Received: 2 February 2015 / Accepted: 4 March 2015 / Published: 12 March 2015

\begin{abstract}
We have built a new isonucleoside derivative on a 2,6-dioxobicyclo[3.2.0]heptane skeleton as a potential anti-HIV agent. To synthesize the target compound, an acetal-protected dihydroxyacetone was first converted to a 2,3-epoxytetrahydrofuran derivative. Introduction of an azide group, followed by the formation of an oxetane ring, gave a pseudosugar derivative with a 2,6-dioxobicyclo[3.2.0]heptane skeleton. The desired isonucleoside was obtained by constructing a purine base moiety on the scaffold, followed by amination.
\end{abstract}

Keywords: nucleoside; bicyclo; oxetane ring; conformation

\section{Introduction}

Since the discovery of 3'-azidothymidine (AZT), much attention has been paid to the development of effective chemotherapeutic agents against the human immunodeficiency virus (HIV), a causative agent for AIDS [1,2]. More than 20 anti-HIV drugs have now been approved and are clinically used for the treatment of AIDS. Among them, nucleoside reverse transcriptase inhibitors (NRTIs) play a 
critical role in the treatment of AIDS patients. In the most successful regimen for AIDS referred to as ART (Anti-Retroviral Therapy), a cocktail of anti-HIV drugs, including NRTIs, non-nucleoside reverse transcriptase inhibitors (NNRTIs), and protease inhibitors (PIs) [3], is used. Although ART greatly contributes to increasing the lifespan of patients, drug-resistant strains of the virus are still a serious problem [4,5]. Therefore, new drugs that are effective against the resistant virus strains are constantly needed.

Most NRTIs belong to a category of dideoxynucleosides, e.g., zalcitabine (ddC) [6] and didanosine (ddI) [6]. AZT [7] and lamivudine [8] are 3'-substituted dideoxynucleoside derivatives, and abacavir is a carbocyclic analogue of dideoxynucleoside. Only tenofovir [9], which is a nucleoside phosphonate (Figures 1 and 2), is different. From the viewpoint of designing new anti-HIV agents, nucleosides constructed on a novel scaffold are expected to have antiviral activity against the resistant virus strains and may avoid cross-resistance to the known NRTIs.<smiles>Cc1cn([C@@H]2CC(C#N)[C@@H](CO)O2)c(=O)[nH]c1=O</smiles>

Zidovudine (AZT)<smiles>Nc1ccn(C2CCC(CO)O2)c(=O)n1</smiles>

Zalcitabine (ddC)<smiles>O=c1[nH]cnc2c1ncn2[C@H]1CC[C@@H](CO)O1</smiles>

Didanosine (ddl)<smiles>C[C@H](Cn1cnc2c(N)ncnc21)OCP(=O)(O)O</smiles>

Tenofovir

Figure 1. Approved NRTIs.<smiles>Cc1cn([C@@H]2C=C[C@@H](CO)O2)c(=O)[nH]c1=O</smiles>

Stavudine (d4T)<smiles>[Y]C1[CH][C@@H]([B])[C@H](CO)C=C1</smiles>

1: $X=O$

2: $\mathrm{X}=\mathrm{CH}_{2}$

3: $X=S$<smiles>OCC1C=CC(n2cnc3c(NC4CC4)ncnc32)C1</smiles>

Abacavir (ABC)

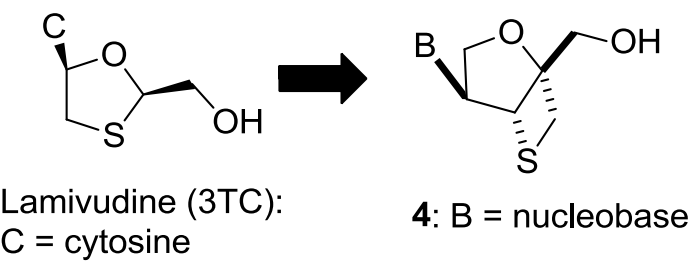

Figure 2. Our previous works searching for anti-HIV nucleosides built on a new scaffold.

Thus, we have been focusing on the design and synthesis of nucleoside derivatives attached to a pseudosugar scaffold [10-17]. Among them, nucleosides with cyclohexenyl [13], dihydrothiophenyl [15], and dihydropyranyl [17] groups in place of a furanose ring have been synthesized as "ring-expanded" analogues of stavudine and abacavir. Dihydropyranyl derivative $\mathbf{1}$ did 
not show any activity, whereas cyclohexenyl derivative 2 showed weak anti-HIV activity [13,17]. On the other hand, dihydrothiophenyl derivative 3 showed significant anti-HIV activity [15]. In addition, we have applied the "ring-expanding" concept to lamivudine and synthesized isonucleosides 4 constructed on 2-oxa-6-thiabicyclo[3.2.0]heptane [14]. The isonucleoside 4 was also considered as conformationally-restricted analogue of lamivudine by introducing a fused thietane ring (vide infra). However, 4 showed no anti-HIV activity (Figure 2). In this study, we planned to build isonucleoside 6 on a 2,6-dioxobicyclo[3.2.0]heptane skeleton, an analogue of dioxolane nucleoside 5 which exhibited potent anti-HIV activity [18-20]. The similar conformationally-restricted analogue of d4T was known: cyclopropane-fused carbocyclic d4T $(N-\mathrm{MCd} 4 \mathrm{~T})$, fixed in north conformation, was originally reported by Marquez and his colleagues and had significant anti-HIV activity with lesser cytotoxicity [21]. In addition, D-enantiomer of 5 was known to have potent cytotoxicity [18-20]. Thus, isonucleoside 6 should be promising although the thietane-fused derivative 4 was inactive (Figure 3).
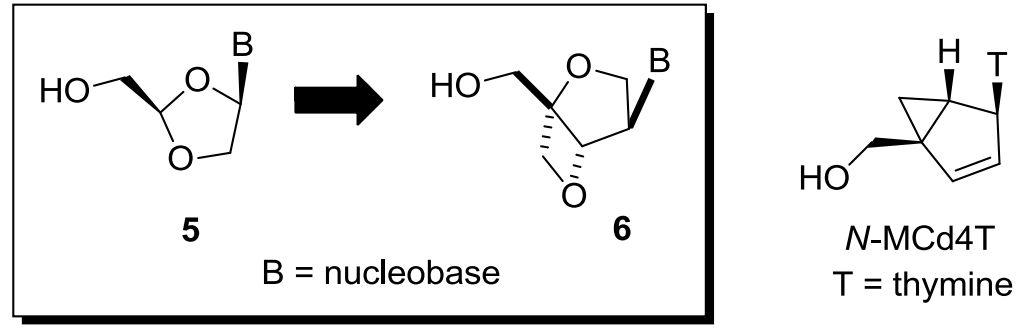

Figure 3. Design of nucleoside derivative built on a 2,6-bicyclo[3.2.0]heptane skeleton.

\section{Results and Discussion}

Following our previous reports [11,14], epoxide 7 was synthesized. We first attempted to introduce an adenine onto 7 by treating it with DBU [22]. However, the reaction did not give the desired product 9 (Scheme 1).<smiles>CC1(C)OCC2(CO1)[C@H](O)[C@H]2O</smiles><smiles>Nc1ncnc2[nH]cnc12</smiles><smiles>CN(C)CC(C)(C)C</smiles><smiles></smiles>

Scheme 1. Attempt to introduce adenine moiety.

In addition, Lewis acid-catalyzed reactions did not afford 9 either (data not shown). Since the low reactivity of 7 might be due to its rigid structure, we next tried nucleophilic substitution using a more reactive cyclic sulfate derivative [23]. Cis-allyl alcohol 10, a precursor of epoxide 7 [11,14], was cyclized under Mitsunobu conditions, as in the case of epoxide 7 [11,14], to give dihydrofuran 11 in $71 \%$ yield. Treatment of dihydrofuran 11 with potassium osmate in the presence of $N$-methylmorpholine $N$-oxide afforded cis-diol 12. The desired cyclic sulfate 13 was obtained by treatment of 12 with thionyl chloride, followed by oxidation. However, the nucleophilic substitution of 13 with adenine did not afford the desired isonucleoside 14 (Scheme 2). 


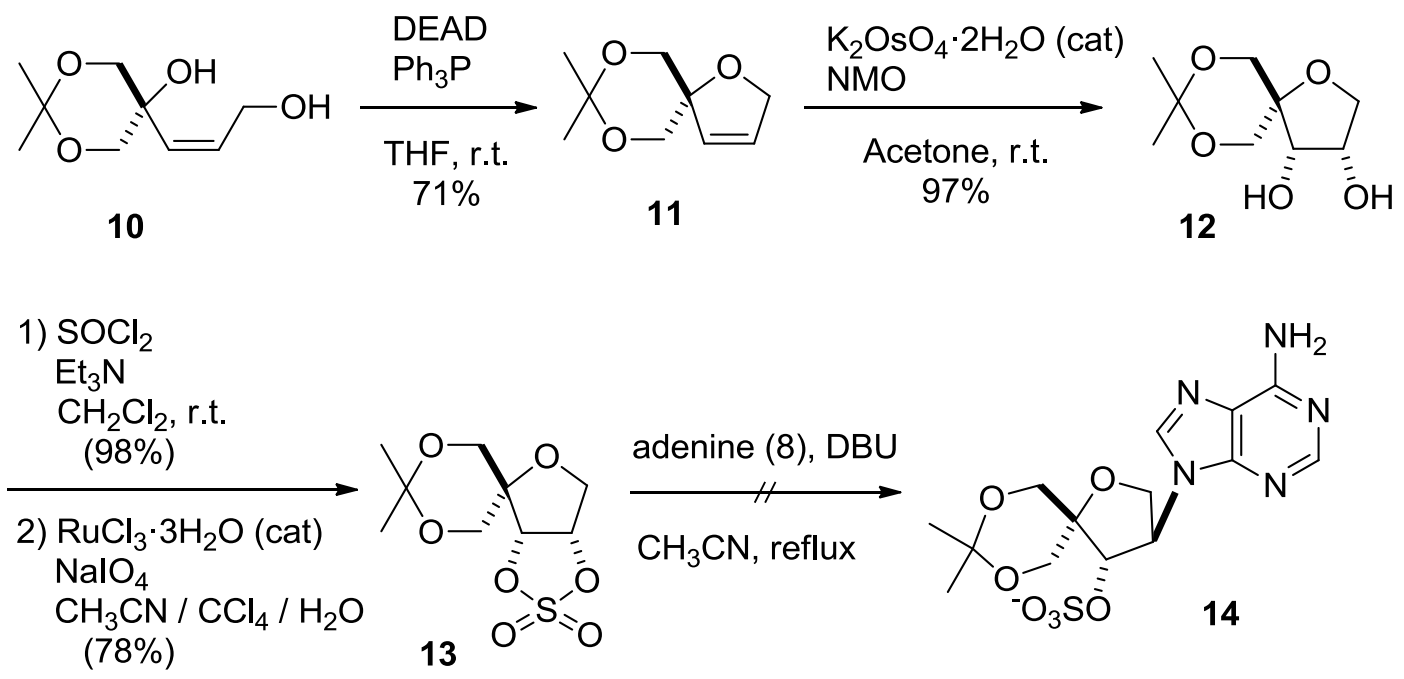

Scheme 2. Second attempt to introduce adenine using a cyclic sulfate.

Therefore, we revised our plan to synthesize an isoadenosine constructed on a 2,6-dioxobicyclo[3.2.0]heptane scaffold, and the revised scheme is shown in Scheme 3 in a retrosynthetic manner. Instead of the direct introduction of adenine, we decided to build the adenine ring on the 2,6-dioxobicyclo[3.2.0]heptane pseudosugar skeleton in a stepwise manner. According to this plan, compound $\mathbf{1 6}$ was thought to be a suitable intermediate for preparing $\mathbf{1 5}$ since it can be transformed to $\mathbf{6}$ by the formation of an imidazole ring, followed by amination. Fused oxetane derivative 16 can be obtained from dimesylate 17. Finally, epoxide 7, described above, was selected as the starting compound because it can be converted to $\mathbf{1 7}$ by the selective cleavage of the oxirane ring with an azide anion (Scheme 2).<smiles>Nc1ncnc2c1ncn2C1OC2(CO)COC12</smiles>

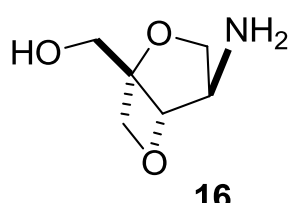
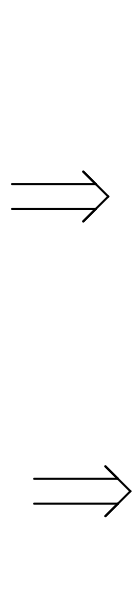<smiles>Nc1c(Cl)ncnc1NC1O[C@@]2(CO)COC12</smiles>

15

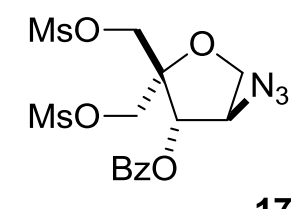

17
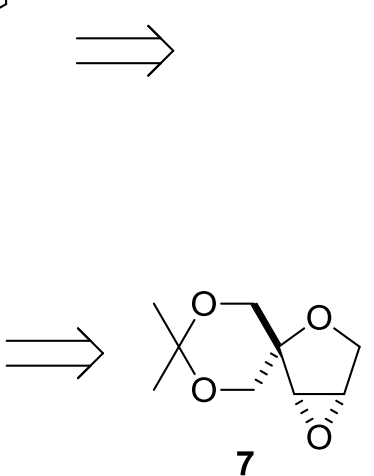

7

Scheme 3. Revised retrosynthesis of isoadenosine 6 built on a 2,6-dioxobicyclo[3.2.0]heptane skeleton.

First, regioselective cleavage of the oxirane ring of 7 with sodium azide in 2-methoxyethanol under reflux conditions gave the desired azide-alcohol 18 as a single regioisomer in $66 \%$ yield. It is obvious that the nucleophilic azide anion attacked from the less hindered side since similar regioselective epoxide opening was observed in our previous report [11,14]. After benzoylation of the hydroxyl group, the acetal group of $\mathbf{1 9}$ was removed by using acidic hydrolysis, and the resulting diol was 
mesylated to give dimesylate $\mathbf{2 0}$ in good yield. Deprotection of the benzoyl group and the subsequent formation of an oxetane ring were achieved by treating 20 with sodium methoxide under reflux conditions to give mesylate $\mathbf{2 1}$ in $72 \%$ yield. The structure of $\mathbf{2 1}$ was unambiguously determined by comparison of 1D NMR spectrum with that of 2-oxa-6-thiabicyclo[3.2.0]heptane skeleton [14] after converting it to benzoate 22 by treatment with benzoic acid in the presence of cesium fluoride. In ${ }^{1} \mathrm{H}-\mathrm{NMR}$ spectra of 22, the peaks corresponding to the methyl groups of the dimesylate were absent, and only the peaks corresponding to the benzoyl group in the range of 8.1-7.4 ppm were present. In addition, one of the methylene protons at the 2-position was observed as a doublet at $4.42 \mathrm{ppm}$, meaning that the coupling with H-3 disappeared. This indicates that the conformation around the tetrahydrofuran ring changes and becomes fixed, which causes a loss of coupling between one pair of $\mathrm{H}-2$ and $\mathrm{H}-3$ protons. A similar correlation between conformation and couplings in ${ }^{1} \mathrm{H}-\mathrm{NMR}$ spectra has been reported for the 2-oxa-6-thiabicyclo[3.2.0]heptane skeleton [14]. Moreover, in the mass spectrum of the compound, a molecular ion peak was observed at $\mathrm{m} / \mathrm{z}=276$, further supporting the assignment of the structure. Finally, 22 was deprotected to afford azido-alcohol 23 in $88 \%$ yield (Scheme 4).
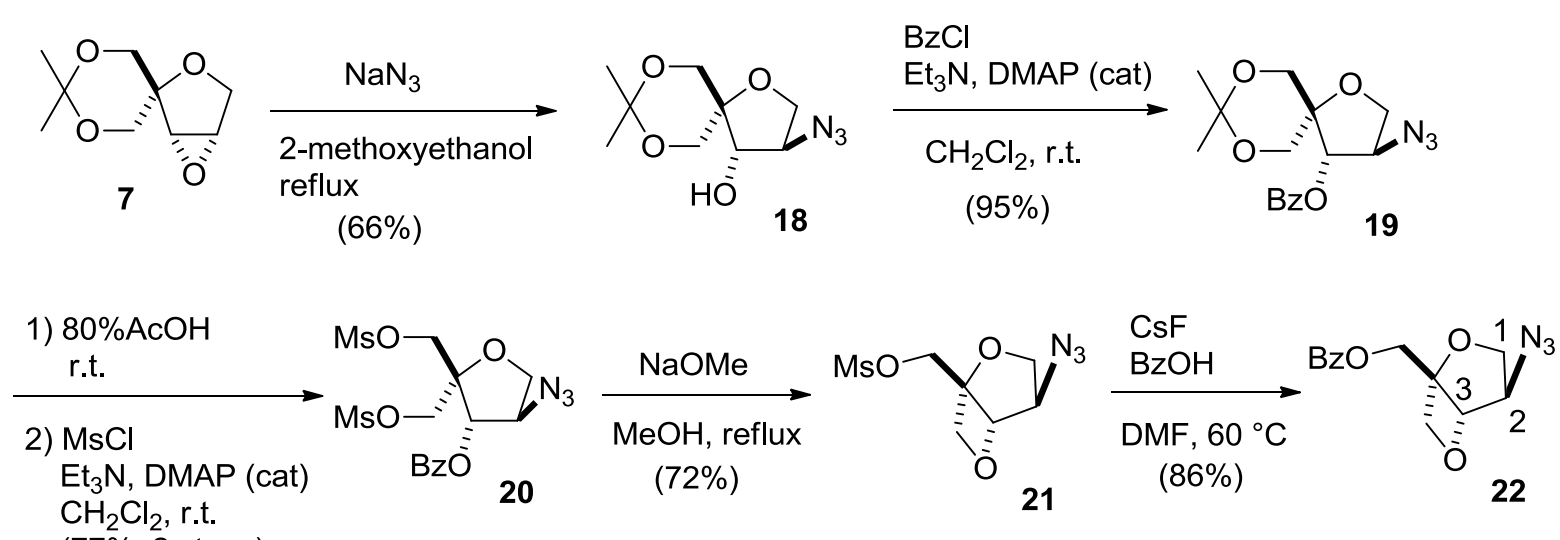
$(77 \%, 2$ steps)
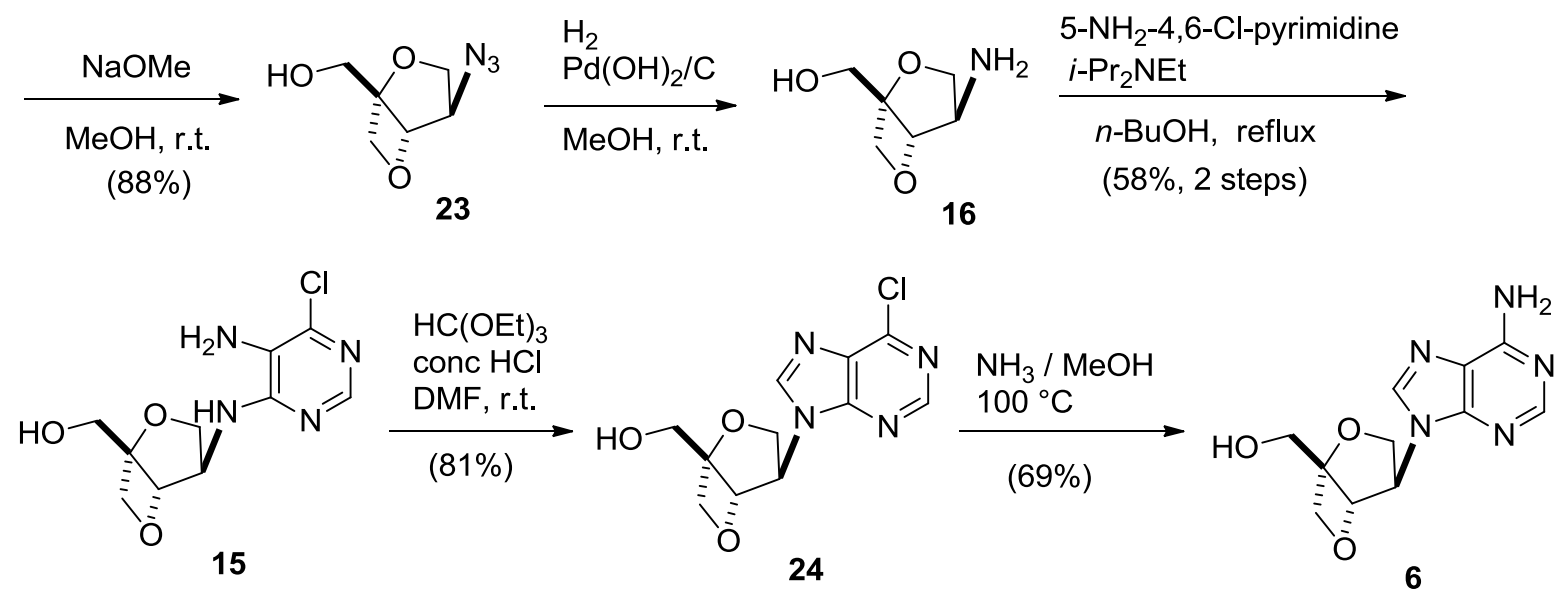

Scheme 4. Synthesis of isoadenosine 6.

Azido-alcohol 23 was reduced by catalytic hydrogenation to give key intermediate 16, which was treated with 5-amino-4,6-dichloropyrimidine and diisopropylethylamine in refluxing $n$-butanol [23] to give diaminopyrimidine derivative $\mathbf{1 5}$ in 58\% yield from 23. Formation of the imidazole ring of 15 was 
accomplished by treatment with orthoethyl formate under acidic conditions [24] to give 6-chloropurine nucleoside 24. Finally, the isoadenosine was built on the 2,6-dioxobicyclo[3.2.0] heptane scaffold 6 by heating 24 with methanolic ammonia in a sealed tube in 69\% yield (Scheme 4). Isoadenosine 6 did not show any significant activity against HIV even at a concentration of $100 \mu \mathrm{M}$.

\section{Experimental Section}

\section{General Information}

Melting points are uncorrected. NMR spectra were recorded at $400 \mathrm{MHz}\left({ }^{1} \mathrm{H}\right), 100 \mathrm{MHz}\left({ }^{13} \mathrm{C}\right)$ using $\mathrm{CDCl}_{3}$ as a solvent. As an internal standard, tetramethylsilane was used for $\mathrm{CDCl}_{3}$. Mass spectra were obtained by EI or FAB mode. Silica gel for chromatography was Silica Gel 60N (spherical, neutral, 100-210 $\mu \mathrm{m}$, Kanto Chemical Co. Inc., Tokyo, Japan). When the reagents sensitive to moisture were used, the reaction was performed under argon atmosphere.

8,8-Dimethyl-1,7,9-trioxaspiro[4,5]dec-3-ene (11). To a solution of $\mathrm{PPh}_{3}$ (2.49 g, $\left.9.48 \mathrm{mmol}\right)$ in THF $(10 \mathrm{~mL})$ was added DEAD $(4.31 \mathrm{~mL}, 9.48 \mathrm{mmol})$ and the mixture was stirred at room temperature for $5 \mathrm{~min}$. To this mixture, a solution of $\mathbf{1 0}[11,14](1.04 \mathrm{~g}, 5.58 \mathrm{mmol})$ in THF $(10 \mathrm{~mL})$ was added. The mixture was stirred at room temperature for $1 \mathrm{~h}$. After the solvent was removed under reduced pressure, the residue was purified by silica gel column chromatography (hexane-ethyl acetate $=19: 1$ ) to give 11 (677 mg, 71\%) as a white crystal. mp 47-49 ${ }^{\circ} \mathrm{C} ;{ }^{1} \mathrm{H}-\mathrm{NMR}\left(\mathrm{CDCl}_{3}\right) \delta 1.45(3 \mathrm{H}, \mathrm{s}), 1.48(3 \mathrm{H}$, s), $3.76(2 \mathrm{H}, \mathrm{d}, J=11.6 \mathrm{~Hz}), 3.81(2 \mathrm{H}, \mathrm{d}, J=11.6 \mathrm{~Hz}), 4.71(2 \mathrm{H}, \mathrm{t}, J=1.9 \mathrm{~Hz}), 5.84-5.87(1 \mathrm{H}, \mathrm{m})$, $6.02(1 \mathrm{H}, \mathrm{d}, J=6.3 \mathrm{~Hz}) ;{ }^{13} \mathrm{C}-\mathrm{NMR}\left(\mathrm{CDCl}_{3}\right) \delta 22.6,24.6,66.7,74.9,98.0,128.1,129.1$; IR (neat) 2924.2, 2853.1, 1724.1, 1215.9, $758.3 \mathrm{~cm}^{-1}$; FAB-MS (m/z) $155[\mathrm{M}-15]^{+}$; Anal. Calcd for $\mathrm{C}_{9} \mathrm{H}_{14} \mathrm{O}_{3}$; C, 62.84; H, 8.32. Found; C, 62.78; H, 8.44.

(3S*,4S*)-8,8-Dimethyl-1,7,9,-trioxaspiro[4.5]decane-3,4-diol (12). To a solution of 11 (73 $\mathrm{mg}$, $0.43 \mathrm{mmol})$ and $\mathrm{NMO}(0.22 \mathrm{~mL})$ in acetone $(4 \mathrm{~mL})$, was added a solution of $\mathrm{K}_{2} \mathrm{OsO}_{4} \cdot 2 \mathrm{H}_{2} \mathrm{O}(1 \mathrm{mg}$, $0.004 \mathrm{mmol})$ in $\mathrm{H}_{2} \mathrm{O}(0.4 \mathrm{~mL})$ at $0{ }^{\circ} \mathrm{C}$. After stirred at room temperature for $60 \mathrm{~h}, \mathrm{Na}_{2} \mathrm{~S}_{2} \mathrm{O}_{3} \cdot 5 \mathrm{H}_{2} \mathrm{O}$ $(125 \mathrm{mg})$ was added and the mixture was stirred at room temperature for $30 \mathrm{~min}$. After the whole mixture was dried over $\mathrm{Na}_{2} \mathrm{SO}_{4}$, the solid materials were removed by suction and washed with ethyl acetate. The combined filtrate was concentrated under reduced pressure. The residue was purified by silica gel column chromatography $\left(\mathrm{CHCl}_{3}-\mathrm{MeOH}=19: 1\right)$ to give $12(84 \mathrm{mg}, 97 \%) .{ }^{1} \mathrm{H}-\mathrm{NMR}\left(\mathrm{CDCl}_{3}\right)$ $\delta 1.43(3 \mathrm{H}, \mathrm{s}), 1.49(3 \mathrm{H}, \mathrm{s}), 3.59(1 \mathrm{H}, \mathrm{dd}, J=11.6,1.9 \mathrm{~Hz}), 3.80(1 \mathrm{H}, \mathrm{d}, J=9.7 \mathrm{~Hz}), 3.82-3.87(2 \mathrm{H}$, m), $3.94(1 \mathrm{H}, \mathrm{dd}, J=9.7,4.9 \mathrm{~Hz}), 4.14(1 \mathrm{H}, \mathrm{dd}, J=11.6,1.9 \mathrm{~Hz}), 4.22(1 \mathrm{H}, \mathrm{d}, J=5.3 \mathrm{~Hz}) 4.34(1 \mathrm{H}, \mathrm{q}$, $J=5.0 \mathrm{~Hz}) ;{ }^{13} \mathrm{C}-\mathrm{NMR}\left(\mathrm{CDCl}_{3}\right) \delta 21.0,25.8,63.3,66.5,71.0,71.4,74.9,76.7,98.4$; IR $(\mathrm{KBr}) 3306.4$, 2953.6, 2741.6, 1452.2, $524.19 \mathrm{~cm}^{-1}$; EI-MS $(\mathrm{m} / \mathrm{z}): 204[\mathrm{M}+1]^{+}$; HRMS Calcd for $\mathrm{C}_{9} \mathrm{H}_{15} \mathrm{~N}_{3} \mathrm{O}_{4}$ : 204.0998, Found: 204.0992.

(3S*,4S*)-8,8-Dimethyl-1,7,9,-trioxaspiro[4.5]decane-3,4-cyclicsulfate (13). To a solution of 12 (410 mg, $2.01 \mathrm{mmol})$ and $\mathrm{Et}_{3} \mathrm{~N}(672 \mu \mathrm{L}, 4.82 \mathrm{mmol})$ in $\mathrm{CH}_{2} \mathrm{Cl}_{2}(10 \mathrm{~mL})$, was added dropwise a solution of $\mathrm{SOCl}_{2}(113 \mu \mathrm{L}, 1.55 \mathrm{mmoL})$ in $\mathrm{CH}_{2} \mathrm{Cl}_{2}(10 \mathrm{~mL})$ at $0{ }^{\circ} \mathrm{C}$. After stirred at room temperature for $15 \mathrm{~min}$, the mixture was washed with water. The water layer was extracted with $\mathrm{CHCl}_{3}$ twice and the combined 
organic layer was washed with brine, then dried over $\mathrm{Na}_{2} \mathrm{SO}_{4}$. After filtration, the residue was passed through a short silica gel column (eluate: hexane-ethyl acetate $=1: 1$ ). After the solvents were removed under reduced pressure, the residue was dissolved in $\mathrm{CCl}_{4}-\mathrm{CH}_{3} \mathrm{CN}-\mathrm{H}_{2} \mathrm{O}(2: 2: 3,3 \mathrm{~mL})$. To this solution, were added $\mathrm{RuCl}_{3} \cdot 3 \mathrm{H}_{2} \mathrm{O}(2.7 \mathrm{mg})$ and $\mathrm{NaIO}_{4}(73 \mathrm{mg}, 0.34 \mathrm{mmol})$ at $0{ }^{\circ} \mathrm{C}$. The mixture was stirred at the same temperature for $1.5 \mathrm{~h}$. After diluted with ether, the mixture was washed with water, sat. $\mathrm{NaHCO}_{3}$ and brine, then dried over $\mathrm{Na}_{2} \mathrm{SO}_{4}$. After filtration, the solvents were removed under reduce pressure, the residue was purified by silica gel column chromatography (hexane-ethyl acetate $=6: 1$ ) to give $13(82 \mathrm{mg}, 77 \%) .{ }^{1} \mathrm{H}-\mathrm{NMR}\left(\mathrm{CDCl}_{3}\right) \delta 1.43(3 \mathrm{H}, \mathrm{s}), 1.50(3 \mathrm{H}, \mathrm{s}), 3.56(1 \mathrm{H}, \mathrm{dd}, J=12.1,2.4 \mathrm{~Hz})$, $3.82(1 \mathrm{H}, \mathrm{d}, J=12.1 \mathrm{~Hz}), 3.91(1 \mathrm{H}, \mathrm{d}, J=12.1 \mathrm{~Hz}), 3.97(1 \mathrm{H}, \mathrm{dd}, J=12.6,4.4 \mathrm{~Hz}), 4.06(1 \mathrm{H}, \mathrm{dd}$, $J=12.1,2.4 \mathrm{~Hz}), 4.28(1 \mathrm{H}, \mathrm{d}, J=12.6 \mathrm{~Hz}), 5.42(1 \mathrm{H}, \mathrm{d}, J=6.3 \mathrm{~Hz}), 5.48(1 \mathrm{H}, \mathrm{t}, J=5.1 \mathrm{~Hz}) ;{ }^{13} \mathrm{C}-\mathrm{NMR}$ $\left(\mathrm{CDCl}_{3}\right) \delta 19.6,27.1,61.0,62.4,69.7,79.0,83.3,84.5,99.2$; IR (KBr) 3000.8, 2892.3, 1699.7, 1380.9, $1089.9 \mathrm{~cm}^{-1}$; EI-MS (m/z): 267 [M+1] ; HRMS Calcd for $\mathrm{C}_{9} \mathrm{H}_{15} \mathrm{~N}_{3} \mathrm{O}_{4}: 266.0460$, Found: 266.0467.

$\left(3 R^{*}, 4 S^{*}\right)$-3-Azido-8,8-dimethyl-1,7,9,-trioxaspiro[4.5]decan-4-ol (18). A mixture of 7 [11,14] (433 mg, $2.33 \mathrm{mmol})$ and $\mathrm{NaN}_{3}(752 \mathrm{mg} 11.6 \mathrm{mmol})$ in 2-methoxyethanol $(26 \mathrm{~mL})$ was kept at $100{ }^{\circ} \mathrm{C}$ for $5 \mathrm{~h}$. After the solvent was removed under reduced pressure, the residue was dissolved in ethyl acetate. After washed with water and brine, the organic layer was dried over $\mathrm{Na}_{2} \mathrm{SO}_{4}$. After filtration, the solvents were removed under reduce pressure, the residue was purified by silica gel column chromatography (hexane-ethyl acetate $=5: 1)$ to give $18(351 \mathrm{mg}, 66 \%) .{ }^{1} \mathrm{H}-\mathrm{NMR}\left(\mathrm{CDCl}_{3}\right) \delta 1.40(3 \mathrm{H}$, s), $1.49(3 \mathrm{H}, \mathrm{s}), 3.69-3.80(3 \mathrm{H}, \mathrm{m}), 3.84(1 \mathrm{H}, \mathrm{d}, J=11.6 \mathrm{~Hz}), 4.01-4.08(3 \mathrm{H}, \mathrm{m}), 4.38(1 \mathrm{H}, \mathrm{t}, J=1.9$, $2.4 \mathrm{~Hz}) ;{ }^{13} \mathrm{C}-\mathrm{NMR}\left(\mathrm{CDCl}_{3}\right) \delta 19.5,27.4,62.3,66.0,67.6,69.4,78.2,79.1,98.5$; IR (neat) 3419.0, 2104.8, 1086.6, $831.7 \mathrm{~cm}^{-1}$; EI-MS $(\mathrm{m} / z)$ : $229[\mathrm{M}+1]^{+}$; HRMS Calcd for $\mathrm{C}_{9} \mathrm{H}_{15} \mathrm{~N}_{3} \mathrm{O}_{4}: 229.1063$, Found: 229.1062.

(3R*,4S*)-3-Azido-8,8-dimethyl-1,7,9,-trioxaspiro[4.5]decan-4-yl benzoate (19). To a solution of 18 (432 mg, $1.88 \mathrm{mmol}), \mathrm{Et}_{3} \mathrm{~N}(0.59 \mathrm{~mL}, 4.24 \mathrm{mmol})$, and DMAP (23 mg, $\left.0.19 \mathrm{mmol}\right)$ in $\mathrm{CH}_{2} \mathrm{Cl}_{2}(15 \mathrm{~mL})$ was added benzoyl chloride $(0.40 \mathrm{~mL}, 3.39 \mathrm{mmol})$ and the mixture was stirred at room temperature for $6.5 \mathrm{~h}$. The reaction was quenched by addition of $\mathrm{MeOH}$, and the whole was stirred at room temperature for $10 \mathrm{~min}$. The mixture was diluted with $\mathrm{CH}_{2} \mathrm{Cl}_{2}$ and washed with water and brine, then dried over $\mathrm{Na}_{2} \mathrm{SO}_{4}$. After filtration, the solvents were removed under reduce pressure, the residue was purified by silica gel column chromatography (hexane-ethyl acetate $=4: 1)$ to give $\mathbf{1 9}(598 \mathrm{mg}, 95 \%)$. ${ }^{1} \mathrm{H}-\mathrm{NMR}\left(\mathrm{CDCl}_{3}\right) \delta 1.37(3 \mathrm{H}, \mathrm{s}), 1.47(3 \mathrm{H}, \mathrm{s}), 3.84-3.91(3 \mathrm{H}, \mathrm{m}), 3.99(1 \mathrm{H}, \mathrm{dd}, J=1.4,10.6 \mathrm{~Hz}), 4.07$ $(1 \mathrm{H}, \mathrm{dd}, J=1.4,10.6 \mathrm{~Hz}), 4.18-4.25(2 \mathrm{H}, \mathrm{m}) 5.47(1 \mathrm{H}, \mathrm{d}, J=1.0 \mathrm{~Hz}), 7.48(2 \mathrm{H}, \mathrm{t}, J=7.2 \mathrm{~Hz}), 7.62$ $(1 \mathrm{H}, J=7.2 \mathrm{~Hz}), 8.03(2 \mathrm{H}, J=7.2 \mathrm{~Hz}) ;{ }^{13} \mathrm{C}-\mathrm{NMR}\left(\mathrm{CDCl}_{3}\right) \delta$ 22.4, 24.2, 62.1, 65.0, 66.2, 69.9, 78.9, 79.2, 98.4, 128.6, 129.0, 129.6, 133.6, 165.3; IR (neat) 2993.1, 2107.2, 1725.4, 1267.4, 1091.3, 711.5 $\mathrm{cm}^{-1}$; EI-MS $(\mathrm{m} / z): 333$ [M] $]^{+}$; HRMS Calcd for $\mathrm{C}_{16} \mathrm{H}_{19} \mathrm{~N}_{3} \mathrm{O}_{5}: 333.1325$, Found: 333.1336.

(3S*,4R*)-4-Azido-2,2-bis((methylsulfonyloxy)methyl)tetrahydrohuran-3-yl benzoate (20). A mixture of $19(1.01 \mathrm{~g}, 3.04 \mathrm{mmol})$ in $80 \% \mathrm{AcOH}(80 \mathrm{~mL})$ was stirred at room temperature for $5 \mathrm{~h}$. After the solvent was removed under reduced pressure, the residue was co-evaporated with EtOH five times to remove residual $\mathrm{AcOH}$. The resulting crude product was dissolved in $\mathrm{CH}_{2} \mathrm{Cl}_{2}(40 \mathrm{~mL})$. To this mixture, were added, $\mathrm{MsCl}(1.19 \mathrm{~mL}, 15.18 \mathrm{mmol}), \mathrm{Et}_{3} \mathrm{~N}(2.14 \mathrm{~mL}, 15.18 \mathrm{mmol})$, and DMAP (38 mg, 
$0.30 \mathrm{mmol}$ ). After stirred at room temperature for $1 \mathrm{~h}$, the mixture was diluted with $\mathrm{CH}_{2} \mathrm{Cl}_{2}$ washed with $5 \% \mathrm{HCl}$, sat. $\mathrm{NaHCO}_{3}$ and brine. The separated organic layer was dried over $\mathrm{Na}_{2} \mathrm{SO}_{4}$. After filtration, the solvents were removed under reduce pressure, the residue was purified by silica gel column chromatography (hexane-ethyl acetate $=2: 1)$ to give $20(1.05 \mathrm{~g}, 77 \%) .{ }^{1} \mathrm{H}-\mathrm{NMR}\left(\mathrm{CDCl}_{3}\right) \delta$ $3.00(3 \mathrm{H}, \mathrm{s}), 3.12(3 \mathrm{H}, \mathrm{s}), 3.94(1 \mathrm{H}, \mathrm{dd}, J=5.80,4.37 \mathrm{~Hz}), 4.33 \sim 4.48(6 \mathrm{H}, \mathrm{m}), 5.48(1 \mathrm{H}, \mathrm{d}$, $J=3.4 \mathrm{~Hz}), 7.50(2 \mathrm{H}, \mathrm{t}, J=7.7 \mathrm{~Hz}), 7.64(1 \mathrm{H}, \mathrm{t}, J=8.0 \mathrm{~Hz}), 8.04(2 \mathrm{H}, \mathrm{d}, J=7.3 \mathrm{~Hz}) ;{ }^{13} \mathrm{C}-\mathrm{NMR}$ $\left(\mathrm{CDCl}_{3}\right) \delta 37.7,65.5,65.8,67.4,70.3,77.2,78.9,82.8,128.1,128.8,129.9,134.2,165.2$; IR (near) 2110.7, 1728.8, 1360.0, $1267.0 \mathrm{~cm}^{-1}$; FAB-MS $(\mathrm{m} / \mathrm{z}): 450[\mathrm{M}+1]^{+}$; HRMS Calcd for $\mathrm{C}_{15} \mathrm{H}_{20} \mathrm{~N}_{3} \mathrm{O}_{9} \mathrm{~S}_{2}$ : 450.0641, Found: 450.0631.

$\left(\left(1 R^{*}, 4 R^{*}, 5 S^{*}\right)-4\right.$-Azido-2,6-dioxabicyclo[3.2.0] heptan-1-yl)methyl methanesulfonate (21). A mixture of 20 (36 mg, $0.08 \mathrm{mmol})$ and $\mathrm{NaOCH}_{3}(4.6 \mathrm{mg}, 0.08 \mathrm{mmol})$ in $\mathrm{MeOH}(2 \mathrm{~mL})$ was kept at $75{ }^{\circ} \mathrm{C}$ overnight. After the solvent was removed under reduced pressure, the residue was dissolved in $\mathrm{CHCl}_{3}$ and washed with water and brine, then dried over $\mathrm{Na}_{2} \mathrm{SO}_{4}$. After filtration, the solvents were removed under reduce pressure, the residue was purified by silica gel column chromatography (hexane-ethyl acetate $=2: 1)$ to give $21(14 \mathrm{mg}, 72 \%) .{ }^{1} \mathrm{H}-\mathrm{NMR}\left(\mathrm{CDCl}_{3}\right) \delta 3.09(3 \mathrm{H}, \mathrm{s}), 4.05(1 \mathrm{H}, \mathrm{d}, J=3.4 \mathrm{~Hz}), 3.40$ $(1 \mathrm{H}, \mathrm{d}, J=11.1 \mathrm{~Hz}), 4.29 \sim 4.77(4 \mathrm{H}, \mathrm{m}), 4.76(1 \mathrm{H}, \mathrm{d}, J=8.2 \mathrm{~Hz}), 5.06(1 \mathrm{H}, \mathrm{s}) ;{ }^{13} \mathrm{C}-\mathrm{NMR}\left(\mathrm{CDCl}_{3}\right) \delta$ 37.8, 63.6, 67.4, 71.9, 77.1, 84.4, 89.9; IR (neat) 2098.2, 1360.4, 1175.0, 960.2, $815.5 \mathrm{~cm}^{-1}$; FAB-MS $(\mathrm{m} / \mathrm{z}): 250[\mathrm{M}+1]^{+}$; HRMS Calcd for $\mathrm{C}_{7} \mathrm{H}_{12} \mathrm{~N}_{3} \mathrm{O}_{5} \mathrm{~S}: 250.0498$, Found: 250.0490 .

$\left(\left(1 R^{*}, 4 R^{*}, 5 S^{*}\right)-4\right.$-Azido-2,6-dioxabicyclo[3.2.0]heptan-1-yl)methyl benzoate (22). A mixture of CsF (332 mg, $2.19 \mathrm{mmol}$ ) and PhCOOH (267 mg, $2.19 \mathrm{mmol}$ ) in DMF (40 mL) was stirred at room temperature for $20 \mathrm{~min}$. To this mixture was added a solution of 21 (182 $\mathrm{mg}, 0.73 \mathrm{mmol}$ ) in DMF $(20 \mathrm{~mL})$. After stirred at $60{ }^{\circ} \mathrm{C}$ overnight, the mixture was partitioned between EtOAc and $\mathrm{H}_{2} \mathrm{O}$. The separated water layer was extracted with EtOAc, and the organic layer was washed with sat. $\mathrm{NaHCO}_{3}$, brine, then dried over $\mathrm{Na}_{2} \mathrm{SO}_{4}$. After filtration, the filtrated was concentrated under reduced pressure. The residue was purified by silica gel column chromatography (hexane-ethyl acetate $=5: 1$ ) to give 22 (170 mg, 86\%). ${ }^{1} \mathrm{H}-\mathrm{NMR}\left(\mathrm{CDCl}_{3}\right) \delta 4.08(1 \mathrm{H}, \mathrm{d}, J=3.4 \mathrm{~Hz}), 4.42(1 \mathrm{H}, \mathrm{d}, J=10.6 \mathrm{~Hz}), 4.52(2 \mathrm{H}, \mathrm{dd}$, $J=10.6,3.4 \mathrm{~Hz}), 4.60(2 \mathrm{H}, \mathrm{s}), 4.83(1 \mathrm{H}, \mathrm{d}, J=7.7 \mathrm{~Hz}), 5.15(1 \mathrm{H}, \mathrm{s}), 7.61(2 \mathrm{H}, \mathrm{t}, J=15.5 \mathrm{~Hz}) 7.59$ $(1 \mathrm{H}, \mathrm{t}, J=17.4 \mathrm{~Hz}), 8.10(2 \mathrm{H}, \mathrm{d}, J=9.7 \mathrm{~Hz}) ;{ }^{13} \mathrm{C}-\mathrm{NMR}\left(\mathrm{CDCl}_{3}\right) \delta 63.1,63.8,71.5,77.8,85.1,90.4$, 128.4, 129.4, 129.7, 133.3, 166.1; IR (neat) 2099.7, 1723.1, 1284.3, $713.4 \mathrm{~cm}^{-1}$; FAB-MS (m/z); $276[\mathrm{M}+1]^{+}$; HRMS Calcd for $\mathrm{C}_{13} \mathrm{H}_{14} \mathrm{~N}_{3} \mathrm{O}_{4}: 276.0984$, Found: 276.0977.

$\left(\left(1 S^{*}, 4 R^{*}, 5 S^{*}\right)-4-A z i d o-2,6-d i o x a-b i c y c l o[3.2 .0]\right.$ heptan-1-yl)methanol (23). A mixture of 22 (184 mg, $0.67 \mathrm{mmol})$ and $\mathrm{NaOCH}_{3}(19 \mathrm{mg}, 0.33 \mathrm{mmol})$ in $\mathrm{MeOH}(15 \mathrm{~mL})$ was stirred at room temperature. After the mixture was neutralized with $\mathrm{AcOH}(19 \mu \mathrm{L})$, the solvents were removed under reduced pressure and the residue was purified by silica gel column chromatography (hexane-ethyl acetate $=4: 1$ ) to give 23 (90 mg, 79\%). ${ }^{1} \mathrm{H}-\mathrm{NMR}\left(\mathrm{CDCl}_{3}\right) \delta 3.91(2 \mathrm{H}, \mathrm{d}, J=6.3 \mathrm{~Hz}), 4.04(1 \mathrm{H}, \mathrm{d}, J=3.9 \mathrm{~Hz}), 4.36$ $(1 \mathrm{H}, \mathrm{d}, J=10.6 \mathrm{~Hz}), 4.48(2 \mathrm{H}, \mathrm{dd}, J=10.6,3.4 \mathrm{~Hz}), 4.48(1 \mathrm{H}, \mathrm{d}, J=8.2 \mathrm{~Hz}), 4.68(1 \mathrm{H}, \mathrm{d}, J=8.2 \mathrm{~Hz})$, $5.05(1 \mathrm{H}, \mathrm{s}) ;{ }^{13} \mathrm{C}-\mathrm{NMR}\left(\mathrm{CDCl}_{3}\right) \delta 62.3,64.0,71.5,77.6,86.9,90.2$; IR (neat) 3431.5, 2101.6, 1248.2, $870.88 \mathrm{~cm}^{-1}$; EI-MS $(\mathrm{m} / z): 171[\mathrm{M}]^{+}$; HRMS Calcd for $\mathrm{C}_{6} \mathrm{H}_{9} \mathrm{~N}_{3} \mathrm{O}_{3}: 171.0644$, Found: 171.0643. 
((1S*,4R*,5S*)-4-(5-Amino-6-chloropyrimidin-4-ylamino)-2,6-dioxa-bicyclo[3.2.0]heptan-1-yl)methanol (15). A mixture of $23(69 \mathrm{mg}, 0.40 \mathrm{mmol})$ and $\mathrm{Pd}(\mathrm{OH})_{2}(6.2 \mathrm{mg}, 0.04 \mathrm{mmol})$ in $\mathrm{MeOH}(5 \mathrm{~mL})$ was stirred at room temperature overnight under $\mathrm{H}_{2}$ atmosphere. After insoluble materials were removed by filtration, the solvents were removed under reduced pressure. The resulting crude product was dissolved in $n$-BuOH (3 mL). To this mixture, were added 5-amino-4,6-dichloropyrimidine (140.1 mg, $0.86 \mathrm{mmol})$ and $i$ - $\operatorname{Pr}_{2} \mathrm{NEt}(298 \mu \mathrm{L}, 1.71 \mathrm{mmol})$. The mixture was kept under reflux overnight. After the solvents were removed under reduced pressure, the residue was purified by silica gel column chromatography (chloroform-methanol $=19: 1)$ to give $15(64 \mathrm{mg}, 58 \%) .{ }^{1} \mathrm{H}-\mathrm{NMR}\left(\mathrm{CD}_{3} \mathrm{OD}\right) \delta 3.67$ $(2 \mathrm{H}, \mathrm{q}, J=12.6 \mathrm{~Hz}), 4.24(1 \mathrm{H}, \mathrm{d}, J=10.1 \mathrm{~Hz}), 4.38(1 \mathrm{H}, \mathrm{d}, J=7.7 \mathrm{~Hz}), 4.45(1 \mathrm{H}, \mathrm{d}, J=4.4 \mathrm{~Hz}), 4.50$ $(1 \mathrm{H}, \mathrm{d}, J=10.1,4.4 \mathrm{~Hz}), 4.63(1 \mathrm{H}, \mathrm{d}, J=7.2 \mathrm{~Hz}) 4.90(1 \mathrm{H}, \mathrm{s}), 7.72(1 \mathrm{H}, \mathrm{s}) ;{ }^{13} \mathrm{C} \mathrm{NMR}\left(\mathrm{CD}_{3} \mathrm{OD}\right) \delta$ 58.1, 58.3, 62.7, 73.1, 88.3, 92.1, 125.5, 138.9, 147.3, 153.3; IR (KBr) 3381.3, 2926.7, 1578.6, $1056.3 \mathrm{~cm}^{-1}$; EI-MS (m/z): $272[\mathrm{M}]^{+}$; HRMS Calcd for $\mathrm{C}_{10} \mathrm{H}_{13} \mathrm{ClN}_{4} \mathrm{O}_{3}: 272.0676$, Found: 272.0673.

$\left(\left(1 S^{*}, 4 R^{*}, 5 S^{*}\right)-4-(6-C h l o r o-9 H-p u r i n-9-y l)-2,6-d i o x a-b i c y c l o[3.2 .0]\right.$ heptan-1-yl)methanol (24). To a solution of $15(18 \mathrm{mg}, 0.07 \mathrm{mmol})$ in $\operatorname{DMF}(0.5 \mathrm{~mL})$, were added orthoethyl formate $(0.7 \mathrm{~mL}$, $4.21 \mathrm{mmol})$ and conc $\mathrm{HCl}(2 \mu \mathrm{L}, 0.024 \mathrm{mmol})$ at $0{ }^{\circ} \mathrm{C}$. After the mixture was stirred at room temperature, the solvents were removed under reduced pressure. The residue was dissolved in $0.5 \mathrm{M}$ $a q \mathrm{HCl}(1 \mathrm{~mL})$ and the mixture was stirred at room temperature for $1 \mathrm{~h}$. The mixture was neutralized with $1 \mathrm{M}$ aqNaOH $(0.5 \mathrm{~mL})$ and concentrated under reduced pressure. The residue was extracted with a solution of chloroform-methanol $=1: 1$. After the insoluble materials were removed by filtration, the solvents were removed under reduced pressure. The residue was purified by $p$ TLC (developed by chloroform-methanol $=5: 1)$ to give $24(14.9 \mathrm{mg}, 81 \%) .{ }^{1} \mathrm{H}-\mathrm{NMR}\left(\mathrm{CDCl}_{3}\right) \delta 4.12(2 \mathrm{H}, \mathrm{q}, J=12.6 \mathrm{~Hz})$, $4.59(1 \mathrm{H}, \mathrm{d}, J=11.6 \mathrm{~Hz}), 4.66(1 \mathrm{H}, \mathrm{d}, J=9.7 \mathrm{~Hz}), 4.68(1 \mathrm{H}, \mathrm{d}, J=9.7 \mathrm{~Hz}), 4.66(1 \mathrm{H}, \mathrm{d}, J=9.7 \mathrm{~Hz})$, $4.90(1 \mathrm{H}, \mathrm{dd}, J=11.1,4.8 \mathrm{~Hz}), 5.24(1 \mathrm{H}, \mathrm{s}), 5.38(1 \mathrm{H}, \mathrm{d}, J=4.4 \mathrm{~Hz}), 8.68(1 \mathrm{H}, \mathrm{s}), 8.76(1 \mathrm{H}, \mathrm{s})$; ${ }^{13} \mathrm{C}-\mathrm{NMR}\left(\mathrm{CDCl}_{3}-\mathrm{CD}_{3} \mathrm{OD}=19: 1\right) \delta 58.6,61.2,71.9,77.2,87.8,90.9,130.7,144.6,150.7,151.2$, 151.8; IR (KBr) 3401.4, 2931.1, 1597.4, 1567.4, 1056.6 $\mathrm{cm}^{-1}$; EI-MS (m/z): $282[\mathrm{M}]^{+}$; HRMS Calcd for $\mathrm{C}_{11} \mathrm{H}_{11} \mathrm{ClN}_{4} \mathrm{O}_{3}$ : 282.0520, Found: 282.0506 .

$\left(\left(1 S^{*}, 4 R^{*}, 5 S^{*}\right)-4-(6-A m i n o-9 H-p u r i n-9-y l)-2,6-d i o x a-b i c y c l o[3.2 .0]\right.$ heptan-1-yl)methanol

Compound $24(29.4 \mathrm{mg}, 0.10 \mathrm{mmol})$ was dissolved in sat. methanolic ammonia $(7 \mathrm{~mL})$ and the mixture was kept at $100{ }^{\circ} \mathrm{C}$ for $21 \mathrm{~h}$ in a glass sealed tube. After the solvents were removed under reduced pressure, the residue was purified by $p$ TLC (developed by chloroform-methanol $=5: 1$ ) to give $6(18.8 \mathrm{mg}, 69 \%) .{ }^{1} \mathrm{H}-\mathrm{NMR}\left(\mathrm{CDCl}_{3}-\mathrm{CD}_{3} \mathrm{OD}=17: 3\right) \delta 3.91(1 \mathrm{H}, \mathrm{d}, J=12.6 \mathrm{~Hz}), 4.00(1 \mathrm{H}, \mathrm{d}$, $J=12.1 \mathrm{~Hz}), 4.57(1 \mathrm{H}, \mathrm{d}, J=10.6 \mathrm{~Hz}), 4.64(1 \mathrm{H}, \mathrm{d}, J=7.7 \mathrm{~Hz}), 4.70(1 \mathrm{H}, \mathrm{d}, J=7.7 \mathrm{~Hz}), 4.87(1 \mathrm{H}$, $\mathrm{dd}, J=11.1,4.8 \mathrm{~Hz}), 5.19(1 \mathrm{H}, \mathrm{s}), 5.25(1 \mathrm{H}, \mathrm{d}, J=4.8 \mathrm{~Hz}), 8.25(1 \mathrm{H}, \mathrm{s}), 8.28(1 \mathrm{H}, \mathrm{s}) ;{ }^{13} \mathrm{C}-\mathrm{NMR}$ $\left(\mathrm{CDCl}_{3}-\mathrm{CD}_{3} \mathrm{OD}=17: 3\right) \delta 29.5,58.3,61.3,72.1,87.6,91.0,118.2,139.1,148.9,152.6,155.3$; IR (KBr) 3192.2, 2409.9, 1660.5, 1615.0, $1054.9 \mathrm{~cm}^{-1}$; EI-MS $(\mathrm{m} / \mathrm{z}): 263[\mathrm{M}]^{+}$; HRMS Calcd for $\mathrm{C}_{11} \mathrm{H}_{13} \mathrm{~N}_{5} \mathrm{O}_{3}: 263.1018$, Found: 263.1021.

\section{Conclusions}

We constructed an isoadenosine derivative on a 2,6-dioxobicyclo[3.2.0]heptane scaffold. Since our initial attempt to synthesize $\mathbf{6}$ by directly introducing the adenine moiety was not successful, we 
synthesized it by the de novo synthesis of an adenine ring on a pseudosugar moiety. However, this unique adenosine analogue showed no activity against HIV. Previously, we have reported that neither thymine nor adenine analogues 4 built on a 2-oxa-6-thiabicyclo[3.2.0]heptane skeleton inhibit HIV [14]. The structural rigidities of these analogues and isoadenosine 6 due to the introduction of fused thietane and oxetane rings, respectively, appear to inhibit anti-HIV activity. In particular, phosphorylation at the 5'-hydroxyl group would be inhibited since deoxynucleoside kinase recognizes the puckering of sugars [25]. Thus, we are currently preparing new substituted nucleoside derivatives based on $\mathbf{4}$ and $\mathbf{6}$, and the results will be reported elsewhere.

\section{Acknowledgments}

This work was supported in part by a Grant-in-Aid for Scientific Research (No. 24590143, Y.Y.) from JSPS and by a grant of Strategic Research Foundation Grant-aided Project for Private Universities from Ministry of Education, Culture, Sport, Science, and Technology, Japan (MEXT), 2010-2014.

\section{Author Contributions}

YY and TI designed research; SK, HK and TS performed the synthesis of compounds and TI assayed anti-HIV activity. YY wrote the paper. All authors read and approved the final manuscript.

\section{Conflicts of Interest}

The authors declare no conflict of interest.

\section{References}

1. Cihlar, T.; Ray, A.S. Nucleoside and nucleotide HIV reverse transcriptase inhibitors: 25 years after zidovudine. Antivir. Res. 2010, 85, 39-58.

2. Mehellou, Y.; de Clercq, E. Twenty-six years of anti-HIV drug discovery: Where do we stand and where do we go? J. Med. Chem. 2010, 53, 521-538.

3. WHO. Consolidated Guidelines on the Use of Antiretroviral Drugs for Treating and Preventing HIV Infection. Available online: http://www.who.int/hiv/pub/guidelines/arv2013/download/en/ (accessed on 30 June 2013).

4. Meadows, D.C.; Gervey-Hague, J. Current developments in HIV chemotherapy. ChemMedChem 2006, 1, 16-29.

5. Imamichi, T. Action of anti-HIV drugs and resistance: Reverse transcriptase inhibitors and protease inhibitors. Curr. Pharm. Des. 2004, 10, 4039-4053.

6. Mitsuya, H.; Broder, S. Inhibition of the in vitro infectivity and cytopathic effect of human T-lymphotrophic virus type III/lymphadenopathy-associated virus (HTLV-III/LAV) by 2',3'-dideoxynucleosides. Proc. Natl. Acad. Sci. USA 1986, 83, 1911-1915.

7. Mitsuya, H.; Weinhold, K.J.; Furman, P.A.; St Clair, M.H.; Lehrman, S.N.; Gallo, R.C.; Bolognesi, D.; Barry, D.W.; Broder, S. 3'-Azido-3'-deoxythymidine (BW A509U): An antiviral agent that inhibits the infectivity and cytopathic effect of human T-lymphotropic virus type III/lymphadenopathy-associated virus in vitro. Proc. Natl. Acad. Sci. USA 1985, 82, 7096-7100. 
8. Schinazi, R.F.; Chu, C.K.; Peck, A.; McMillan, A.; Mathis, R.; Cannon, D.; Jeong, L.S.; Beach, J.W.; Choi, W.B.; Yeola, S.; et al. Activities of the four optical isomers of 2',3'-dideoxy3'-thiacytidine (BCH-189) against human immunodeficiency virus type 1 in human lymphocytes. Antimicrob. Agents Chemother. 1992, 36, 672-676.

9. Balzarini, J.; Holy, A.; Jindrich, J.; Naesens, L.; Snoeck, R.; Schols, D.; de Clercq, E. Differential antiherpesvirus and antiretrovirus effects of the $(S)$ and $(R)$ enantiomers of acyclic nucleoside phosphonates: Potent and selective in vitro and in vivo antiretrovirus activities of (R)-9-(2-phosphonomethoxypropyl)-2,6-diaminopurine. Antimicrob. Agents Chemother. 1993, 37, 332-338.

10. Yoshimura, Y.; Yamazaki, Y.; Kawahata, M.; Yamaguchi, K.; Takahata, H. Design and synthesis of a novel ring-expanded 4'-Thio-apio-nucleoside derivatives. Tetrahedron Lett. 2007, 48, 4519-4522.

11. Yoshimura, Y.; Asami, K.; Matsui, H.; Tanaka, H.; Takahata, H. New synthesis of ( \pm )-isonucleosides. Org. Lett. 2006, 8, 6015-6018.

12. Yoshimura, Y.; Yamazaki, Y.; Saito, Y.; Takahata, H. Synthesis of 1-(5,6-dihydro-2H-thiopyran2-yl)uracil by a Pummerer-type thioglycosylation reaction: The regioselectivity of allylic substitution. Tetrahedron 2009, 65, 9091-9102.

13. Yoshimura, Y.; Ohta, M.; Imahori, T.; Imamichi, T.; Takahata, H. A new entry to carbocyclic nucleosides: Oxidative coupling reaction of cycloalkenylsilanes with a nucleobase mediated by hypervalent iodine reagent. Org. Lett. 2008, 10, 3449-3452.

14. Yoshimura, Y.; Asami, K.; Imamichi, T.; Okuda, T.; Shiraki, K.; Takahata, H. Design and synthesis of isonucleosides constructed on a 2-oxa-6-thiabicyclo[3.2.0]heptane scaffold. J. Org. Chem. 2010, 75, 4161-4171.

15. Yoshimura, Y.; Yamazaki, Y.; Saito, Y.; Natori, Y.; Imamichi, T.; Takahata, H. Synthesis of 5-thiodidehydropyranylcytosine derivatives as potential anti-HIV agents. Bioorg. Med. Chem. Lett. 2011, 21, 3313-3316.

16. Kiran, Y.B.; Wakamatsu, H.; Natori, Y.; Takahata, H.; Yoshimura, Y. Design and synthesis of a nucleoside and a phosphonate analogue constructed on a branched-threo-tetrofuranose skeleton. Tetrahedron Lett. 2013, 54, 3949-3952.

17. Kan-no, H.; Saito, Y.; Omoto, S.; Minato, S.; Wakamatsu, H.; Natori, Y.; Imamichi, T.; Takahata, H.; Yoshimura, Y. Synthesis of a dihydropyranonucleoside using an oxidative glycosylation reaction mediated by hypervalent iodine. Synthesis 2014, 46, 879-886.

18. Chu, C.K.; Ahn, S.K.; Kim, H.O.; Beach, J.W.; Alves, A.J.; Jeong, L.S.; Islam, Q.; van Roey, P.; Schinazi, R.F. Asymmetric synthesis of enantiomerically pure (-)-(1'R,4'R)-dioxolane-thymine and its anti-HIV activity. Tetrahedron Lett. 1991, 32, 3791-3794.

19. Kim, H.O.; Schinazi, R.F.; Nampalli, S.; Shanmuganathan, K.; Cannon, D.L.; Alves, A.J.; Jeong, L.S.; Beach, J.W.; Chu, C.K. 1,3-dioxolanylpurine nucleosides $(2 R, 4 R)$ and $(2 R, 4 S)$ with selective anti-HIV-1 activity in human lymphocytes. J. Med. Chem. 1993, 36, 30-37.

20. Kim, H.O.; Schinazi, R.F.; Shanmuganathan, K.; Jeong, L.S., Beach, J.W.; Nampalli, S.; Cannon, D.L.; Chu, C.K. L-beta-(2S,4S)- and L-alpha-(2S,4R)-dioxolanyl nucleosides as potential anti-HIV agents: Asymmetric synthesis and structure-activity relationships. J. Med. Chem. 1993, $36,519-528$. 
21. Choi, Y.; George, C.; Comin, M.J.; Brachi, J.J.; Kim, H.S.; Jacobsen, K.A.; Balzarini, J.; Mitsuya, H.; Boyer, P.L.; Hughes, S.H.; et al. A conformationally locked analogue of the anti-HIV agent stavudine. An important correlation between pseudorotation and maximum amplitude. J. Med. Chem. 2003, 46, 3292-3299.

22. D’Alonzo, D.; van Aerschot, A.; Guaragna, A.; Palumbo, G.; Schepers, G.; Capone, S.; Rozenski, J.; Herdewijn, P. Synthesis and base pairing properties of 1',5'-anhydro-L-hexitol nucleic acids (L-HNA). Chem. Eur. J. 2009, 15, 10121-10131.

23. Bera, S.; Nair, V. A new general synthesis of isomeric nucleosides. Tetrahedron Lett. 2001, 42, 5813-5815.

24. Quadrelli, P.; Piccanello, A.; Mella, M.; Corsaro, A.; Pistarà, V. From cyclopentadiene to isoxazoline-carbocyclic nucleosides: A rapid access to biological molecules through aza-Dielse Alder reactions. Tetrahedron 2008, 64, 3541-3547.

25. Comin, M.J.; Vu, B.C.; Boyer, P.L.; Liao, C.; Hughes, S.H.; Marquez, V.E. D-(+)-isomethanocarbathymidine: A high-affinity substrate for herpes simplex virus 1 thymidine kinase. ChemMedChem 2008, 3, 1129-1134.

Sample Availability: Sample of the final compound is available from the authors. About the other compounds, please contact the authors.

(C) 2015 by the authors; licensee MDPI, Basel, Switzerland. This article is an open access article distributed under the terms and conditions of the Creative Commons Attribution license (http://creativecommons.org/licenses/by/4.0/). 